\title{
Clayey materials from the Sierra de la Demanda Range (Spain): their potential as raw materials for the building ceramics industry
}

\author{
R. ARTIGAS ${ }^{1}, M \cdot \operatorname{RODAS}^{1}$, C. J. SÁNCHEZ ${ }^{2} *$, R. MAS ${ }^{3}, M \cdot$ DONDI $^{4}$ \\ AND J. ARRIBAS ${ }^{5}$ \\ ${ }^{1}$ Crystallography and Mineralogy Department, Geological Sciences Faculty, Complutense University of Madrid, \\ Ciudad Universitaria, E-28040 Madrid, Spain, ${ }^{2}$ Applied Mineralogy Laboratory, Chemical Sciences Faculty, \\ University of Castilla-La Mancha, Avd. Camilo José Cela, E-13071 Ciudad Real, Spain, ${ }^{3}$ Stratigraphy Department, \\ Geological Sciences Faculty, Complutense University of Madrid, Ciudad Universitaria, E-28040 Madrid, Spain, \\ ${ }^{4}$ Institute of Science and Technology for Ceramics, CNR-ISTEC, via Granarolo 64, 48018 Faenza, Italy, and \\ ${ }^{5}$ Petrology Department, Geological Sciences Faculty, Complutense University of Madrid, Ciudad Universitaria, \\ E-28040 Madrid, Spain
}

(Received 9 February 2004; revised 17 June 2004)

\begin{abstract}
This work describes the possible use of thick Early Cretaceous clay deposits, which occur in the southern sector of the Sierra de la Demanda range, as raw materials in the manufacture of ceramic articles. The global mineralogical composition is characterized by high proportions of phyllosilicates and quartz with variable quantities of feldspars, carbonates and hematite. The clay mineralogy differentiates two types of raw materials: illitic clay and kaolinitic-illitic clay. A granulometric distribution in the $2-60 \mu \mathrm{m}$ fraction, good behaviour during the drying stage and acceptable results in firing tests confirmed that most samples can be utilized as raw material in the building ceramics industry. The range of suitable firing temperatures for these materials is $950-1000^{\circ} \mathrm{C}$, a temperature which needs to be raised for samples with a high percentage of kaolinite and quartz. Moreover, other materials with abundant calcite $(20-30 \%)$ are suitable for use as modifiers of some properties or colour.
\end{abstract}

KeYwords: illitic clays, kaolinitic-illitic clays, ceramic properties, fluvial systems, Cameros river basin, Spain.

We have studied the constituent materials of the sedimentary filling of the western section of the Cameros basin (Sierra de la Demanda, Northern Spain). This is the first investigation carried out in the area on the utilization of clay materials in the ceramics industry. Our main aim was to characterize the materials, applying several techniques

* E-mail: carlos.sanchez@uclm.es.

DOI: $10.1180 / 0009855054010153$ and performing experimental ceramic tests in the laboratory in order to deduce if these clays are suitable raw materials for use in the manufacture of ceramic building elements.

To our knowledge, there is no company working in this particular industrial sector in the study area. Therefore, the study itself is pioneering in terms of opening up new economic development prospects for the area.

Before undertaking the ceramics study, a sedimentological and mineralogical characterization of all materials occurring in the basin was 
performed. This characterization enabled us to predict which raw materials would be most suitable for the ceramics industry, and to determine their geographical distribution throughout the basin. The latter aspect allowed us to estimate whether their exploitation would be profitable on the basis of extension and distribution of the various stratigraphical levels and formations.

\section{GEOLOGICAL SETTING}

The Cameros basin (Burgos-Soria-La Rioja) is located in the north-western part of the Cordillera Ibérica (northern Spain) and is bordered by the Tertiary Duero, Ebro and Almazán Depressions (Fig. 1a). It was formed in the context of an interplate rifting process which took place between the Late Jurassic and Early Albian periods (Mas et al., 1993; Guimerá et al., 1995; Salas et al., 2001). It has been interpreted as a syncline basin developed on an extensional ramp dipping southwards and joining two structural terraces on a fault which reaches a depth of several kilometres (Mas et al., 1993; Martín Closas and Alonso Millán, 1998). During the Tertiary Period, Alpine compression produced an inversion of the basin, causing the formation of a large overthrust in the northern sector, on to the Tertiary Ebro Basin, and a system of smaller imbricated overthrusts on to the southern edge of the Duero Basin (Guimerá et al., 1995).

The Cameros basin is divided into Cameros Oriental, or Eastern Cameros sub-basin, with high rates of sedimentation and subsidence, which is affected by low-grade metamorphism and hydrothermal activity (Casquet et al., 1992; AlonsoAzcárate et al., 1995; Barrenechea et al., 1995) and Cameros Occidental, or Western Cameros subbasin, bearing thick levels of lutites under early diagenetic conditions (Arribas et al., 2003).

The studied materials correspond to the sedimentary filling of the western sector of the Cameros basin (Fig. 1b), in which seven depositional sequences have been distinguished (Arribas et al., 2003): SD-1 (Nuestra Señora de Brezales Formation), SD-2 (Jaramillo de la Fuente Formation), SD-3 (Río del Salcedal Formation) all from Titonic-Berriasian; SD-4 (Peñacoba Formation, Valanginian-Hauterivian), SD-5 (Pinilla de los Moros Formation, late Hauterivian-early Barremian), SD-6 (Pantano de la Cuerda del Pozo Formation, Barremian) and SD-7 (Abejar and Castrillo de la Reina Formations, Barremian-late
Aptian). These sequences are equivalent to the seven depositional sequences described by Martín Closas and Alonso-Millán (1998). The uppermost sequences consist of tracks of fining-upward fluvio-alluvial deposits, causing a large recurrence of facies.

\section{MATERIALS AND METHODS}

The mineralogical study was performed on 68 samples corresponding to the lutite and marly levels of the eight stratigraphic sections taken to represent the whole of the basin's sedimentary fill (Fig. 2). The location of these stratigraphic sections is shown in Fig. 1a: three are in the northern sector (TRZ, CTV and MON), three in the central sector (MUR, GAN and GAS) and two in the southern sector (PEN and AHE).

After grinding and homogenizing the materials to $<53 \mu \mathrm{m}$, their global mineralogy was studied by X-ray powder diffraction (XRPD). Carbonates preventing a complete sample suspension were eliminated according to the methodology proposed by Moore and Reynolds (1989). Oriented aggregates (OA) were prepared with both the $<2 \mu \mathrm{m}$ and $2-20 \mu \mathrm{m}$ fractions, separated by sedimentation. These OA were treated thermally $\left(550^{\circ} \mathrm{C}\right.$ for $\left.2 \mathrm{~h}\right)$ and chemically (saturation with ethylene glycol and dimethyl sulphoxide). Semi-quantitative estimations were performed using the main peak of every phase, according to the Schultz (1964) method. The samples were analysed using an X-ray diffractometer (Philips PW 1710), with $\mathrm{Cu}-K \alpha$ radiation, $40 \mathrm{kV}$ and $30 \mathrm{~mA}$, graphite monochromator, $2 \times 10^{3}$ cps sensitivity, a time constant of $1 \mathrm{~s}$, measuring the $2-60^{\circ} 2 \theta$ range in unoriented specimens of both the raw and fired clays and the $2-32^{\circ} 2 \theta$ range in the OA.

Chemical analysis of the principal elements was performed using X-ray fluorescence spectrometry (XRF) (Philips PW-1480) working with a Rh tube at $30 \mathrm{kV}$ and $60 \mathrm{~mA}$.

In structural ceramics, the raw material consists, basically, of a composition enriched in phyllosilicates, with variable contents of quartz and smaller percentages of carbonates, feldspars and oxides. Leaving aside this premise, the results obtained from the sedimentological and mineralogical study show that the units that constitute the depositional sequences 1, 3 and 4 (which are high in carbonate, or of heterolitic nature in terms of the siliciclastic formations) have been ignored for technological studies. 

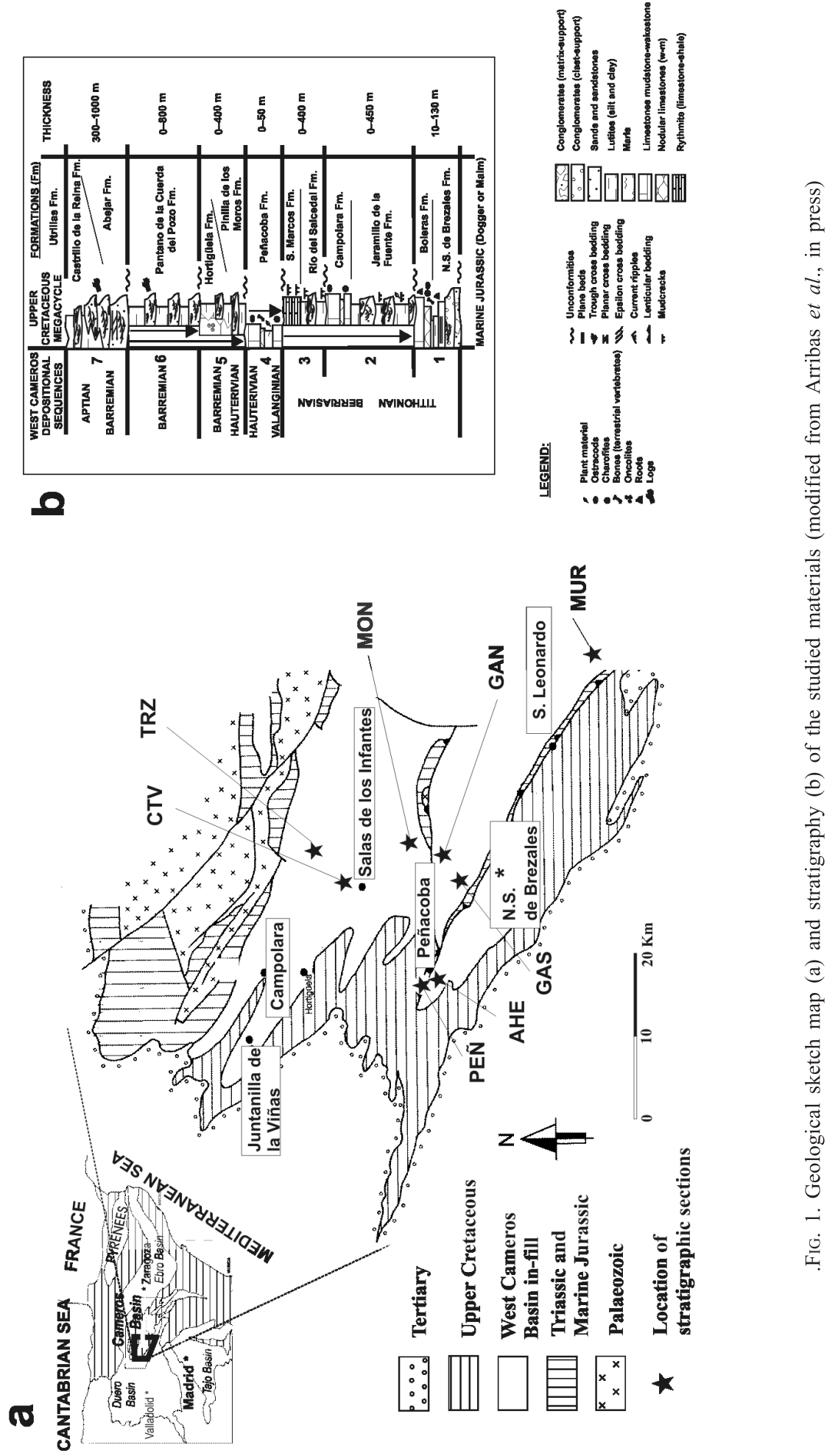


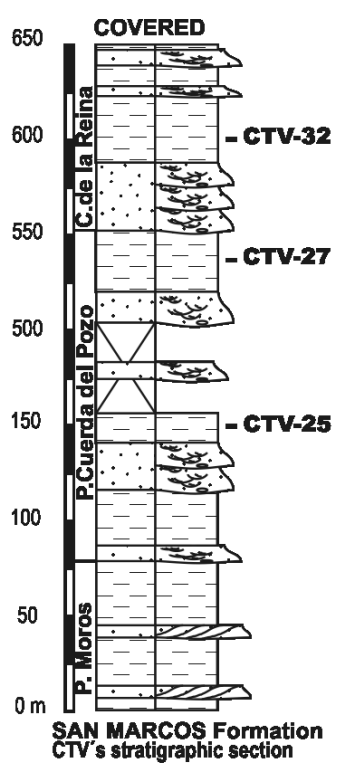

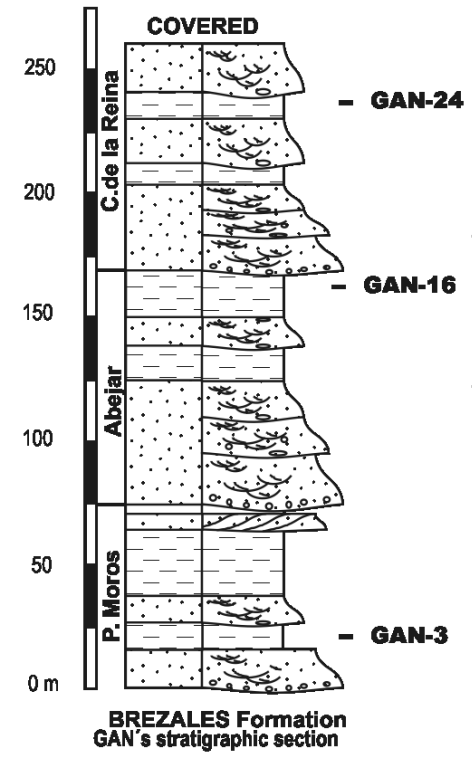

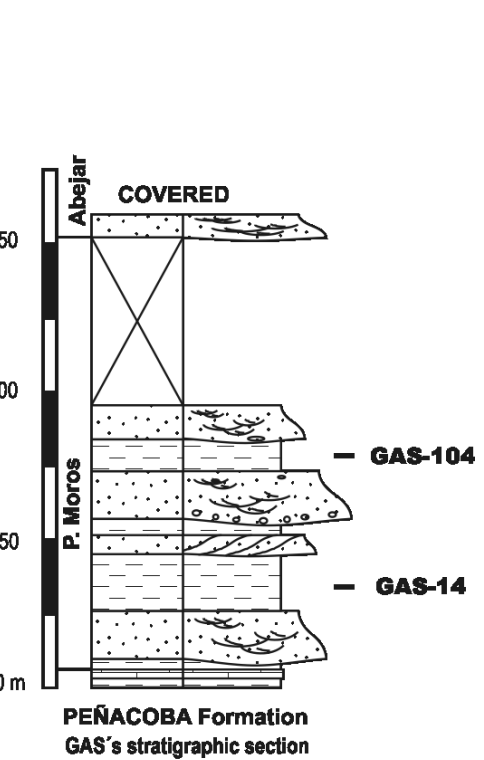

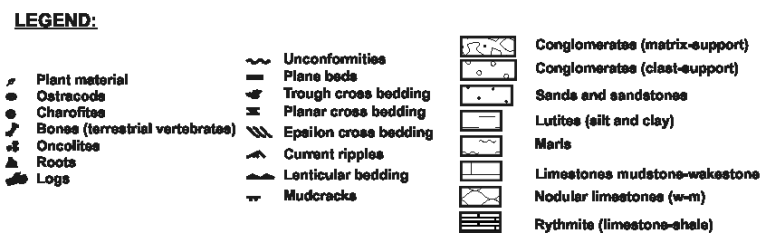

FIG. 2. Stratigraphic sections studied.

Thus the raw materials used in this work are those that constitute the formations of the depositional sequences 2, 5, 6 and 7, because they have the maximum thickness of lutitic levels and are more continuous along the stratigraphical series.

Thirteen representative samples were selected to study the ceramic properties, according to the following criteria: particle-size distribution (Shepard, 1954), thickness of lutitic levels and accessibility of the proposed area.

Granulometric analyses were carried out on the grainsize range $2 \mathrm{~mm}-0.2 \mu \mathrm{m}$. Wet sieving was utilized to measure the larger fraction (NORMA U.N.E. 103-101, 1995) while the fraction below $100 \mu \mathrm{m}$ was analysed by photosedimentation (Micromeritics-Sedigraph 5100 ET). The plasticity was evaluated by the Atterberg limits (liquid and plastic limits, according to the NORMA U.N.E. 103-103, (1994) and NORMA U.N.E. 103-104 (1993) standards, respectively). Thermo-dilatometric analyses were carried out on raw clays using a Netzsch $402 \mathrm{E}$ apparatus $\left(25-1000^{\circ} \mathrm{C}\right.$, heating rate $10^{\circ} \mathrm{C} / \mathrm{min}$ ).
A laboratory simulation of the brickmaking process was conducted through dry grinding (hammer mill), extrusion (pneumatic non-vacuum extruder) of $100 \times 20 \times 10 \mathrm{~mm}$ bars, drying at room temperature $(48 \mathrm{~h})$ and in an oven (overnight), firing in an electric chamber kiln at maximum temperature of 900,950 and $1000^{\circ} \mathrm{C}$, with a heating rate of $3^{\circ} \mathrm{C} / \mathrm{min}$ and soaking time of $4 \mathrm{~h}$. During the drying stage, the Bigot curve, drying shrinkage, hygroscopicity and mechanical resistance (NORMA U.N.E. 67042, 1988) were determined. On fired samples, linear firing shrinkage, water absorption (NORMA UNE 67027, 1984), mechanical resistance (NORMA UNE 67042, 1998), open porosity, apparent density and colour (LANGE Microcolor colourimeter) were determined.

\section{RESULTS}

The siliciclastic formations selected for the technological study were the Pinilla de los Moros (SD-5), Pantano de la Cuerda del Pozo (SD-6), Abejar and Castrillo de la Reina (SD-7) 
because they present the greatest extent and thickness of lutite sediments. The other formations were ignored on the basis of their sedimentological and mineralogical features, since their areal distribution in the basin is very small (SD-2 and SD-3), or their lutite levels are of little economic interest and their mineralogical compositions are too rich in carbonates, therefore unsuitable for use in the manufacture of ceramic products (SD-1 and SD-4).

The Jaramillo de la Fuente Formation (SD-2) exhibits thick levels of lutites and a different source area from the SD-5, 6 and 7 units (Arribas et al., 2001; Arribas et al., 2003); though it is highly heterogenous and present only in the northern sector of the basin, several samples were taken from the TRZ section in order to appraise its suitability as a raw material for the ceramics industry.

\section{Sedimentology of the formations in the Western Cameros Basin}

The stratigraphical sections are shown in Fig. 2 and described as follows:

Jaramillo de la Fuente Formation (SD-2). This unit (up to $400 \mathrm{~m}$ thick) is made up of lenticular bodies of sandstones and silty clays, with minor thin intercalated limestones, passing upwards and laterally into the lacustrine-palustrine facies of the Campolara Formation (maximum thickness $100 \mathrm{~m}$ ).

Pinilla de los Moros Formation (SD-5). This formation is widely distributed and represented throughout the whole basin; it comprises $\sim 400 \mathrm{~m}$ of red silty clays and channellized fluvial sandstone bodies.

Pantano de la Cuerda del Pozo Formation (SD-6). This comprises lenticular sandstone bodies, conglomerate, clay and loam, with small amounts of limestones. The maximum thickness of the unit is $\sim 1,200 \mathrm{~m}$.

Abejar Formation (SD-7). This unit is dominated by sediments of alluvial fan and braided river origin with abundant conglomerate, sands and sandstone bodies.

Castrillo de la Reina Formation (SD-7). These deposits reflect deposition from distal meandering rivers. They include numerous lutite and loam sections, while sandstone intervals are less abundant.

In certain parts of the basin, the last two formations can reach a thickness of $2000 \mathrm{~m}$.

\section{Mineralogy of the siliciclastic formations}

Table 1 shows the results of the mineralogical study of all the siliciclastic formations within the Western Cameros basin, including both the global $(<53 \mu \mathrm{m})$ and the $<2 \mu \mathrm{m}$ fractions.

The Nuestra Señora de Brezales Formation is, along with the Peñacoba Formation, very rich in carbonates, contains no feldspars and has limited amounts of quartz. The Jaramillo de la Fuente unit is richer in phyllosilicates and feldspars, and has a lower carbonate content relative to the other formations. The other units (Río del Salcedal, Pinilla de los Moros, Pantano de la Cuerda del Pozo, Abejar and Castrillo de la Reina) have a similar composition, characterized by high percentages of phyllosilicates and quartz, and minor carbonate, feldspar and hematite (generally $<7 \%$ ).

The small differences in composition are mainly due to carbonates in the MON section and the type of clay minerals. Illite and kaolinite are always predominant, except in the Jaramillo de la Fuente Formation, where illite is associated with minor chlorite concentrations. Randomly interstratified illite-smectite was rarely found in the Pinilla de los Moros and Peñacoba Formations.

The global mineralogy of the different formations filling the western sector of the Cameros Basin is rather homogeneous, showing high phyllosilicate contents $(23-84 \%)$ and quartz $(8-39 \%)$ and lower proportions of feldspars $(<10 \%)$, carbonates $(<15 \%$, except for some samples presenting proportions varying between 20 and 55\%), and hematite $(<8 \%)$. The main variations among the samples are associated with phyllosilicate and carbonate contents (Arribas et al., 2003). In the $<2 \mu \mathrm{m}$ fraction, the most common phyllosilicates are illite $(<80 \%)$ and kaolinite $(<70 \%)$, the former being on average the most abundant. Also present in small proportions are chlorite $(<10 \%)$ and irregular interstratified illite-smectite $(<5 \%)$.

\section{Behaviour in ceramic applications}

Thirteen representative samples were taken from the five formations mentioned above. This section examines the character of the raw materials and their behaviour during shaping, drying and firing.

Characterization of raw materials. The selected samples exhibit a diverse bulk mineralogical composition (Table 1): phyllosilicates (43-76\%), quartz $(13-32 \%)$, calcite $(<7 \%$, except the sample 
TABLE 1. Mineralogical composition of the different siliciclastic formations.

\begin{tabular}{|c|c|c|c|c|c|c|}
\hline & & & & Global fraction & & $<2 \mu \mathrm{m}$ fraction \\
\hline $\begin{array}{l}\text { Depositional } \\
\text { sequence }\end{array}$ & Formation & Sample & Qtz K-Fs & Plg $\mathrm{Cc}$ Dol & Hem Phy & Ill Kln Chl \\
\hline
\end{tabular}

DS-7

\begin{tabular}{|c|c|c|c|c|c|c|c|c|c|c|c|c|}
\hline Castrillo de la & PEÑ-9 & 19 & 10 & $\operatorname{tr}$ & 3 & $\operatorname{tr}$ & $\operatorname{tr}$ & 66 & 51 & 15 & - & - \\
\hline \multirow{13}{*}{ Reina } & PEÑ-7 & 16 & 10 & - & 4 & $\operatorname{tr}$ & $\operatorname{tr}$ & 67 & 57 & 10 & - & - \\
\hline & PEÑ-5* & 19 & 9 & - & 3 & $\operatorname{tr}$ & $\operatorname{tr}$ & 68 & 59 & 9 & - & - \\
\hline & PEÑ-4 & 11 & 4 & $\operatorname{tr}$ & $\operatorname{tr}$ & $\operatorname{tr}$ & $\operatorname{tr}$ & 80 & 72 & 8 & - & - \\
\hline & MON-33 & 13 & $\operatorname{tr}$ & 3 & $\operatorname{tr}$ & $\operatorname{tr}$ & $\operatorname{tr}$ & 80 & 35 & 44 & - & $\operatorname{tr}$ \\
\hline & MON-32 & 16 & $\operatorname{tr}$ & 5 & $\operatorname{tr}$ & $\operatorname{tr}$ & 5 & 69 & 39 & 30 & - & - \\
\hline & CTV-32* & 26 & 3 & 4 & 3 & 3 & $\operatorname{tr}$ & 60 & 43 & 17 & - & - \\
\hline & CTV-31 & 23 & $\operatorname{tr}$ & $\operatorname{tr}$ & $\operatorname{tr}$ & $\operatorname{tr}$ & 5 & 68 & 35 & 31 & $\operatorname{tr}$ & - \\
\hline & CTV-29 & 26 & $\operatorname{tr}$ & $\operatorname{tr}$ & 5 & $\operatorname{tr}$ & 6 & 60 & 43 & 14 & 3 & - \\
\hline & GAN-25 & 24 & 5 & - & 4 & - & $\operatorname{tr}$ & 66 & 41 & 25 & - & - \\
\hline & GAN-24* & 20 & $\operatorname{tr}$ & $\operatorname{tr}$ & $\operatorname{tr}$ & $\operatorname{tr}$ & $\operatorname{tr}$ & 71 & 49 & 22 & - & - \\
\hline & GAN-22 & 22 & 4 & - & $\operatorname{tr}$ & $\operatorname{tr}$ & 5 & 66 & 44 & 22 & - & - \\
\hline & GAN-21 & 23 & $\operatorname{tr}$ & $\operatorname{tr}$ & $\operatorname{tr}$ & - & $\operatorname{tr}$ & 70 & 47 & 23 & - & - \\
\hline & GAN-20 & 24 & 3 & $\operatorname{tr}$ & 4 & 3 & 3 & 62 & 40 & 22 & - & - \\
\hline \multirow[t]{10}{*}{ Abejar } & GAN-17 & 20 & 3 & $\operatorname{tr}$ & 4 & $\operatorname{tr}$ & - & 70 & 35 & 35 & - & - \\
\hline & GAN-16* & 26 & 4 & $\operatorname{tr}$ & 4 & $\operatorname{tr}$ & - & 63 & 38 & 25 & - & - \\
\hline & GAN-15 & 22 & 4 & $\operatorname{tr}$ & 4 & $\operatorname{tr}$ & $\operatorname{tr}$ & 66 & 44 & 22 & - & - \\
\hline & GAN-14 & 24 & 3 & $\operatorname{tr}$ & 3 & $\operatorname{tr}$ & $\operatorname{tr}$ & 65 & 35 & 30 & - & - \\
\hline & GAN-12 & 23 & $\operatorname{tr}$ & $\operatorname{tr}$ & 3 & $\operatorname{tr}$ & - & 70 & 29 & 41 & - & - \\
\hline & GAN-10 & 25 & - & $\operatorname{tr}$ & $\operatorname{tr}$ & - & 4 & 69 & 17 & 52 & - & - \\
\hline & MUR-13 & 24 & $\operatorname{tr}$ & $\operatorname{tr}$ & 3 & $\operatorname{tr}$ & $\operatorname{tr}$ & 67 & 22 & 45 & - & - \\
\hline & MUR-7 & 30 & 4 & $\operatorname{tr}$ & 4 & 3 & $\operatorname{tr}$ & 58 & 19 & 39 & - & - \\
\hline & MUR-4* & 32 & 4 & $\operatorname{tr}$ & 3 & 4 & 4 & 48 & 21 & 27 & - & - \\
\hline & MUR-3 & 30 & $\operatorname{tr}$ & $\operatorname{tr}$ & $\operatorname{tr}$ & $\operatorname{tr}$ & $\operatorname{tr}$ & 64 & 29 & 35 & - & - \\
\hline
\end{tabular}

DS-6

$\begin{array}{llllllllllllll}\text { Pantano de la } & \text { MON-26 } & 12 & 3 & - & 4 & \operatorname{tr} & 3 & 76 & 71 & 5 & - & - \\ \text { Cuerda del Pozo } & \text { MON-23* } & 19 & 4 & 3 & 25 & \operatorname{tr} & 4 & 43 & 37 & 6 & - & - \\ & \text { MON-22 } & 18 & 3 & \operatorname{tr} & 22 & \operatorname{tr} & \operatorname{tr} & 50 & 44 & 6 & - & - \\ & \text { MON-21 } & 14 & \text { tr } & - & \text { tr } & - & \operatorname{tr} & 83 & 46 & 35 & - & \text { tr } \\ & \text { MON-19 } & 16 & \text { tr } & \text { tr } & \text { tr } & \text { tr } & \text { tr } & 80 & 54 & 26 & - & - \\ & \text { MON-18 } & 14 & \text { tr } & - & \text { tr } & \text { tr } & \text { tr } & 79 & 12 & 67 & - & - \\ & \text { MON-17 } & 12 & \text { tr } & - & 25 & \text { tr } & 4 & 55 & 36 & 19 & - & - \\ & \text { CTV-27* } & 25 & \text { tr } & 3 & 4 & 3 & 3 & 59 & 46 & 13 & - & - \\ & \text { CTV-25* } & 22 & 3 & 4 & 4 & 3 & 7 & 56 & 36 & 20 & - & - \\ & \text { CTV-24 } & 15 & \text { tr } & \text { tr } & 3 & 4 & 5 & 69 & 43 & 26 & - & - \\ & \text { CTV-23 } & 16 & \text { tr } & - & \text { tr } & \text { tr } & \text { tr } & 76 & 59 & 17 & - & -\end{array}$

MON-23 with $25 \%)$, dolomite $(<6 \%$, except the sample TRZ-19 with 22\%), K-feldspar $(<10 \%)$, plagioclase $(<6 \%)$, and hematite $(<8 \%)$. The mineralogy of the $<2 \mu \mathrm{m}$ fraction indicates an abundance of illite (36-59\%, except MUR-4 21\%), variable kaolinite content $(0-27 \%)$ and smaller percentages of chlorite and randomly interstratified illite-smectite (5\% in TRZ-19 and COBA-1, respectively). Two groups of raw materials, one mainly illitic (samples: PEÑ-5, MON-23, CTV,27, GAS-104, GAS-14, GAN-3, COBA-1 and TRZ-19), and one illitic- kaolinitic (samples: CTV-32, GAN-24, GAN-16, MUR-4 and CTV-25), containing a kaolinite content $>15 \%$, were established with regard to the mineralogical composition of the clay fraction.

Chemical analysis (Table 2) shows a relative abundance of silica ( $>55 \%)$ and alumina (15-25\%), with an Fe content between 2 and $9 \%$ and a $\mathrm{K}_{2} \mathrm{O}$ content between 2 and $6 \%$, in agreement with the illitic nature of clays. Sample MON-23 is an exception. It presents the largest amount of $\mathrm{CaO}$ $(15 \%)$ and has a loss on ignition of almost $15 \%$. 
Table 1 (contd.)

Global fraction

Depositional Formation Sample Qtz K-Fs Plg Ce Dol Hem Phy Ill Kln Chl I-S sequence

\section{DS-5}

\begin{tabular}{|c|c|c|c|c|c|c|c|c|c|c|c|c|}
\hline Pinilla de los & MON-13 & 11 & 5 & - & 20 & 8 & $\operatorname{tr}$ & 54 & 44 & 8 & $\operatorname{tr}$ & - \\
\hline \multirow{18}{*}{ Moros } & MON-9 & 15 & 4 & - & 16 & 8 & $\operatorname{tr}$ & 55 & 38 & 13 & 4 & - \\
\hline & MON-7 & 16 & 4 & $\operatorname{tr}$ & 17 & 7 & $\operatorname{tr}$ & 53 & 39 & 13 & $\operatorname{tr}$ & - \\
\hline & CTV-20 & 25 & $\operatorname{tr}$ & 3 & $\operatorname{tr}$ & $\operatorname{tr}$ & - & 68 & 38 & 30 & - & - \\
\hline & CTV-16 & 22 & $\operatorname{tr}$ & $\operatorname{tr}$ & $\operatorname{tr}$ & - & 4 & 71 & 50 & 21 & - & - \\
\hline & GAS-104* & 15 & 4 & 4 & 4 & 4 & $\operatorname{tr}$ & 68 & 58 & 10 & - & - \\
\hline & GAS-103 & 15 & $\operatorname{tr}$ & $\operatorname{tr}$ & 5 & $\operatorname{tr}$ & 5 & 70 & 55 & 13 & $\operatorname{tr}$ & - \\
\hline & GAS-101 & 21 & 4 & - & 3 & 3 & $\operatorname{tr}$ & 67 & 51 & 14 & $\operatorname{tr}$ & - \\
\hline & GAS-19 & 13 & 3 & $\operatorname{tr}$ & $\operatorname{tr}$ & 5 & - & 75 & 52 & 23 & - & - \\
\hline & GAS-15 & 27 & 3 & $\operatorname{tr}$ & 3 & $\operatorname{tr}$ & - & 63 & 40 & 23 & - & - \\
\hline & GAS-14* & 25 & 4 & 3 & 4 & $\operatorname{tr}$ & $\operatorname{tr}$ & 61 & 56 & 5 & - & - \\
\hline & GAS-13 & 21 & 3 & $\operatorname{tr}$ & $\operatorname{tr}$ & $\operatorname{tr}$ & $\operatorname{tr}$ & 71 & 60 & 10 & $\operatorname{tr}$ & - \\
\hline & GAS-11 & 20 & 3 & - & 5 & 5 & 3 & 64 & 47 & 15 & $\operatorname{tr}$ & - \\
\hline & GAN-8 & 23 & 7 & - & 5 & $\operatorname{tr}$ & - & 63 & 21 & 42 & - & - \\
\hline & GAN-6 & 24 & $\operatorname{tr}$ & - & $\operatorname{tr}$ & - & 3 & 70 & 24 & 46 & - & - \\
\hline & GAN-4 & 30 & $\operatorname{tr}$ & $\operatorname{tr}$ & $\operatorname{tr}$ & $\operatorname{tr}$ & 3 & 60 & 60 & - & - & - \\
\hline & GAN-3* & 30 & 5 & $\operatorname{tr}$ & 3 & $\operatorname{tr}$ & 3 & 56 & 50 & 6 & - & - \\
\hline & GAN-2 & 26 & - & - & 4 & - & $\operatorname{tr}$ & 69 & 53 & 16 & - & - \\
\hline & COBA-1* & 13 & $\operatorname{tr}$ & $\operatorname{tr}$ & 6 & $\operatorname{tr}$ & $\operatorname{tr}$ & 76 & 58 & 13 & - & 5 \\
\hline
\end{tabular}

DS-4

$\begin{array}{lllllllllllll}\text { Peñacoba } & \text { GAS-10 } & 28 & - & \operatorname{tr} & 16 & 6 & - & 46 & 37 & 6 & 3 & - \\ & \text { GAS-9 } & 25 & - & - & 34 & 9 & \operatorname{tr} & 32 & 24 & 5 & 3 & - \\ & \text { AHE-26 } & 31 & - & - & 23 & - & - & 47 & - & 46 & - & \operatorname{tr} \\ & \text { AHE-16 } & 24 & \operatorname{tr} & - & 43 & - & \operatorname{tr} & 31 & 9 & 18 & - & 4 \\ & \text { AHE-14 } & 32 & - & - & 44 & \operatorname{tr} & - & 23 & 6 & 14 & - & 3\end{array}$

DS-3

$\begin{array}{llrllllllllll}\text { Río del Salcedal } & \text { CTV-10 } & 18 & - & 6 & \operatorname{tr} & \operatorname{tr} & \operatorname{tr} & 72 & 62 & 10 & - & - \\ & \text { CTV-7 } & 18 & \operatorname{tr} & 9 & \operatorname{tr} & - & \operatorname{tr} & 67 & 59 & 8 & - & - \\ & \text { CTV-3 } & 9 & - & \operatorname{tr} & 3 & \operatorname{tr} & 3 & 83 & 77 & 5 & \operatorname{tr} & -\end{array}$

DS-2

$\begin{array}{llrlrrrrrrrrr}\text { Jaramillo } & \text { TRZ-24 } & 8 & \operatorname{tr} & \operatorname{tr} & \operatorname{tr} & 3 & \operatorname{tr} & 84 & 79 & - & 5 & - \\ \text { de la Fuente } & \text { TRZ-21 } & 39 & - & 8 & 12 & \operatorname{tr} & \operatorname{tr} & 39 & 29 & - & 10 & - \\ & \text { TRZ-19* } & 13 & - & 5 & 5 & 22 & 4 & 52 & 47 & - & 5 & - \\ & \text { TRZ-18 } & 12 & - & 3 & 4 & \operatorname{tr} & \operatorname{tr} & 79 & 77 & - & \operatorname{tr} & - \\ & \text { TRZ-12 } & 19 & - & 5 & 13 & - & \operatorname{tr} & 61 & 55 & - & 6 & -\end{array}$

DS-1

$\begin{array}{llllllllllllllll}\text { Sra. de Brezales } & \text { MON-1 } & 19 & \text { tr } & - & 51 & \text { tr } & \text { tr } & 26 & 18 & 8 & -\end{array}$

Symbols (Kretz, 1983): Qtz: quartz, Cc: calcite, Dol: dolomite, Plg: plagioclase, K-Fs: K-feldspar,

Hem: hematite, Phy: phyllosilicates, Ill: illite, Kln: kaolinite, Chl: chlorite, I-S: randomly mixed-layer illitesmectite; tr: traces. The samples marked ${ }^{*}$ are those selected for the study.

Particle-size distribution (Table 3 ) is similar in almost all samples examined. Both the $2-20 \mu \mathrm{m}$ $(47-66 \%)$ and the $<2 \mu \mathrm{m}(20-38 \%)$ fractions predominate over the coarser fractions, which account for minor contributions: $20-60 \mu \mathrm{m}$ $(2-22 \%)$ and $>60 \mu \mathrm{m}(<13 \%)$. Falling outside these ranges are the samples COBA-1 and TRZ19 (50\% and $15 \%<2 \mu \mathrm{m}$, respectively). 
TABLE 2. Chemical composition of raw materials.

\begin{tabular}{lcccccccccrr}
\hline Wt.\% & $\mathrm{SiO}_{2}$ & $\mathrm{Al}_{2} \mathrm{O}_{3}$ & $\mathrm{TiO}_{2}$ & $\mathrm{Fe}_{2} \mathrm{O}_{3}$ & $\mathrm{MnO}$ & $\mathrm{MgO}$ & $\mathrm{CaO}$ & $\mathrm{Na}_{2} \mathrm{O}$ & $\mathrm{K}_{2} \mathrm{O}$ & $\mathrm{P}_{2} \mathrm{O}_{5}$ & LOI \\
\hline PEN-5 & 65.56 & 16.82 & 0.69 & 5.56 & 0.01 & 1.22 & 0.69 & 0.13 & 5.34 & 0.09 & 4.96 \\
CTV-32 & 63.22 & 18.61 & 0.74 & 6.42 & 0.03 & 1.52 & 0.08 & 0.22 & 4.09 & 0.05 & 4.50 \\
GAN-24 & 71.04 & 15.44 & 0.91 & 5.37 & 0.01 & 0.66 & 0.09 & 0.17 & 2.84 & 0.04 & 4.13 \\
GAN-16 & 76.89 & 15.42 & 0.90 & 1.19 & 0.00 & 0.63 & 0.00 & 0.20 & 3.20 & 0.03 & 3.75 \\
MUR-4 & 63.45 & 19.32 & 0.80 & 6.30 & 0.02 & 0.97 & 0.02 & 0.21 & 3.25 & 0.08 & 5.87 \\
MON-23 & 46.21 & 12.42 & 0.66 & 5.39 & 0.18 & 1.77 & 15.04 & 0.54 & 3.29 & 0.07 & 14.63 \\
CTV-27 & 68.12 & 16.28 & 0.73 & 5.09 & 0.02 & 1.43 & 0.17 & 0.18 & 4.16 & 0.08 & 4.19 \\
CTV-25 & 57.51 & 20.68 & 0.75 & 8.55 & 0.07 & 1.37 & 0.94 & 0.25 & 4.29 & 0.08 & 6.30 \\
GAS-104 & 57.58 & 19.21 & 0.80 & 7.20 & 0.03 & 1.80 & 0.39 & 0.24 & 4.82 & 0.08 & 6.99 \\
GAS-14 & 72.41 & 14.87 & 0.71 & 2.98 & 0.03 & 1.88 & 1.58 & 0.15 & 3.37 & 0.05 & 5.94 \\
GAN-3 & 69.86 & 15.38 & 0.68 & 4.85 & 0.03 & 1.39 & 1.22 & 0.15 & 3.74 & 0.07 & 5.54 \\
COBA-1 & 57.64 & 16.02 & 0.83 & 6.05 & 0.05 & 1.55 & 5.15 & 0.40 & 3.92 & 0.06 & 9.69 \\
TRZ-19 & 59.25 & 15.90 & 0.65 & 5.62 & 0.09 & 2.35 & 4.14 & 0.47 & 4.49 & 0.10 & 8.75 \\
& & & & & & & & & & & \\
\hline
\end{tabular}

Total $\mathrm{Fe}$ as $\mathrm{Fe}_{2} \mathrm{O}_{3}$. LOI: Loss on ignition

Shaping behaviour. The behaviour of clays in extrusion was appraised through plasticity measurements (Table 4). Atterberg parameters vary in quite limited ranges: plastic limit $15-24 \%$, liquid limit $25-38 \%$ and plastic index 8-18\%. A single exception was the sample COBA-1, which exhibits very high values of liquid limit $(\sim 65 \%)$ and plastic index $(\sim 41 \%)$, behaviour probably connected with its particularly high phyllosilicates content, including interstratified I-S.

Plotting the data in the plastic limit vs. plastic index diagram, it appears that most samples fulfil the rheological requirements currently accepted in industrial practice (Bain, 1968; Marsigli and Dondi, 1997), though only three clays fall within the optimal field for extrusion, demonstrating that these raw materials are low-plasticity clays (Fig. 3). In contrast, sample COBA-1 is too plastic for extrusion: it could be used in brickmaking to some extent as a plasticizing additive to balance poorly plastic clay materials.

Drying behaviour. The results of the drying tests are summarized in Table 4. Drying shrinkage values are in a rather restricted range (3.4-5.0\%), except on one side, sample COBA-1 has a higher value $(6.9 \%)$ and on the other side samples GAN-16 and TRZ-19 exhibit values of $\sim 2.6 \%$. The different shrinkage shown by the samples referred to is related, to a large extent, to their finer (COBA-1) or coarser particle size (TRZ-19).

TABLE 3. Granulometric distribution of raw materials.

Wt. $\% \quad<2 \mu \mathrm{m} \quad 2-20 \mu \mathrm{m} \quad 20-60 \mu \mathrm{m} \quad 60-200 \mu \mathrm{m} \quad 200-400 \mu \mathrm{m} \quad 400-1000 \mu \mathrm{m} \quad 1000-2000 \mu \mathrm{m} \quad>2 \mathrm{~mm}$

\begin{tabular}{|c|c|c|c|c|c|c|c|c|}
\hline PEÑ-5 & 33 & 49 & 10 & 4 & 3 & 1 & 0 & 0 \\
\hline CTV-32 & 33 & 55 & 8 & 1 & 1 & 1 & 1 & 0 \\
\hline GAN-24 & 38 & 59 & 7 & 2 & 2 & 1 & 1 & 0 \\
\hline GAN-16 & 35 & 52 & 11 & 2 & 0 & 0 & 0 & 0 \\
\hline MUR-4 & 28 & 54 & 12 & 2 & 1 & 1 & 1 & 1 \\
\hline MON-23 & 21 & 62 & 12 & 1 & 1 & 1 & 1 & 1 \\
\hline CTV-27 & 30 & 48 & 12 & 4 & 2 & 2 & 2 & 0 \\
\hline CTV-25 & 26 & 66 & 5 & 1 & 1 & 1 & 0 & 0 \\
\hline GAS-104 & 31 & 66 & 2 & 1 & 0 & 0 & 0 & 0 \\
\hline GAS-14 & 27 & 47 & 22 & 4 & 0 & 0 & 0 & 0 \\
\hline GAN-3 & 20 & 54 & 16 & 3 & 2 & 2 & 2 & 1 \\
\hline COBA-1 & 50 & 42 & 4 & 1 & 1 & 1 & 1 & 0 \\
\hline TRZ-19 & 15 & 69 & 11 & 1 & 1 & 1 & 1 & 1 \\
\hline
\end{tabular}


TABLE 4. Plasticity and drying properties.

\begin{tabular}{lcccccc}
\hline Samples & $\begin{array}{c}\text { Liquid } \\
\text { limit }\end{array}$ & $\begin{array}{c}\text { Atterberg } \\
\text { Plastic } \\
\text { limit }\end{array}$ & $\begin{array}{c}\text { Plasticity } \\
\text { index }\end{array}$ & $\begin{array}{c}\text { Linear } \\
\text { shrinkage (\%) }\end{array}$ & $\begin{array}{c}\text { Drying behaviour } \\
\text { Bending strength } \\
\left(\mathrm{kg} / \mathrm{cm}^{2}\right)\end{array}$ & Hygroscopicity \\
\hline PEN-5 & 30.50 & 18.74 & 11.76 & 4.7 & 30 & 1.17 \\
CTV-32 & 31.70 & 20.65 & 11.05 & 4.1 & 24 & 0.65 \\
GAN-24 & 34.60 & 16.58 & 18.02 & 4.2 & 29 & 0.58 \\
GAN-16 & 34.35 & 19.57 & 14.78 & 2.6 & 12 & 0.32 \\
MUR-4 & 37.35 & 20.15 & 17.20 & 3.4 & 15 & 0.63 \\
MON-23 & 34.15 & 22.75 & 11.40 & 4.0 & 23 & 1.05 \\
CTV-27 & 26.40 & 15.10 & 11.30 & 4.0 & 25 & 1.44 \\
CTV-25 & 33.80 & 22.60 & 11.20 & 4.3 & 22 & 1.41 \\
GAS-104 & 38.15 & 21.80 & 16.35 & 5.0 & 21 & 0.78 \\
GAS-14 & 33.90 & 20.03 & 13.87 & 3.6 & 36 & 0.72 \\
GAN-3 & 27.90 & 19.66 & 8.24 & 6.5 & 87 & 1.70 \\
COBA-1 & 65.40 & 24.30 & 41.10 & 2.7 & 6 & 0.88 \\
TRZ-19 & 25.54 & 18.03 & 7.51 & & & \\
\hline
\end{tabular}

The Bigot curve measurements, obtained using the barellatograph, show that the illitic materials behave in a more uniform way, with respect to the illitickaolinitic ones (Fig. 4). With the initial drying phase carried out at a constant rate, which depends on the temperature-humidity conditions, the slope of the curve is similar in all samples. The most important difference concerns the critical point at which the clay reaches the leatherhard consistency and there is no further shrinkage. When the variations in shrinkage reached their minimum values, the drying process could be accelerated. This point corresponds to water contents as high as $8-15 \%$ with the illitickaolinitic samples having slightly lower values.
However, while most samples exhibit a distinct shoulder, others present a gradual transition to the non-shrinkage drying phase (e.g. GAN-16).

The moisture absorption capacity (hygroscopicity) is generally $<1 \%$, which implies a low humidity reabsorption risk. The average hygroscopicity of illitic clays is somewhat greater than that of illitic-kaolinitic ones $(1.04 \%$ and $0.72 \%$, respectively). The higher values (COBA-1) are clearly related to the occurrence of smectite.

The dry mechanical resistance, bending strength, ranges from 6 to $87 \mathrm{~kg} / \mathrm{cm}^{2}$ (Table 4). The illitickaolinitic clays exhibit slightly lower values $\left(12-29 \mathrm{~kg} / \mathrm{cm}^{2}\right)$ than the illitic ones

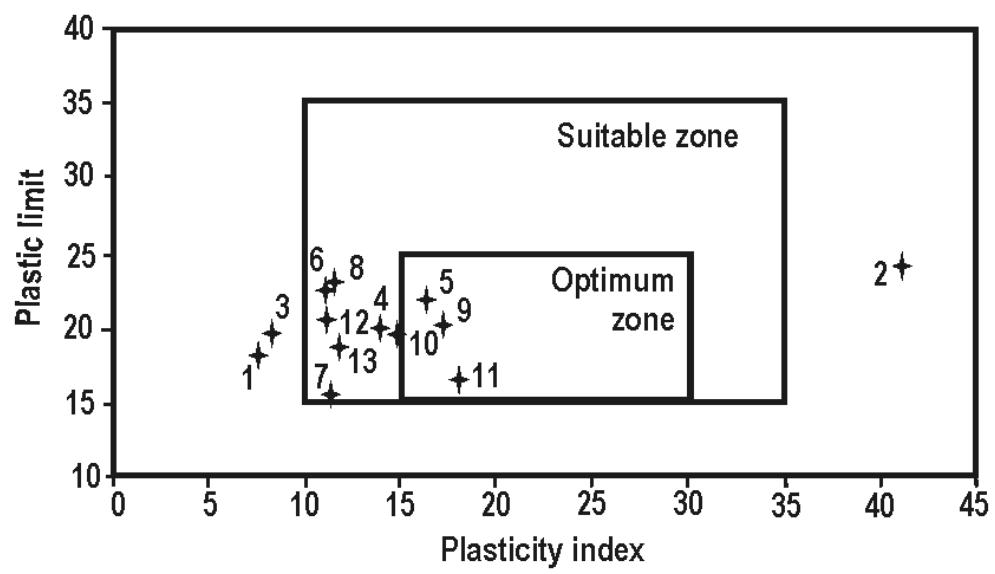

FIG. 3. Prediction of extrusion behaviour on the basis of Atterberg plasticity limits (Bain, 1968; Dondi et al., 1998b). 1: TR2-19; 2: COBA-1; 3: GAN-3; 4: GAS-14; 5: GAS-104; 6: CTV-25; 7: CTV-27; 8: MON-23; 9: MUR-4; 10: GAN-16; 11: GAN-24; 12: CTV-32; 13: PEÑ-5. 

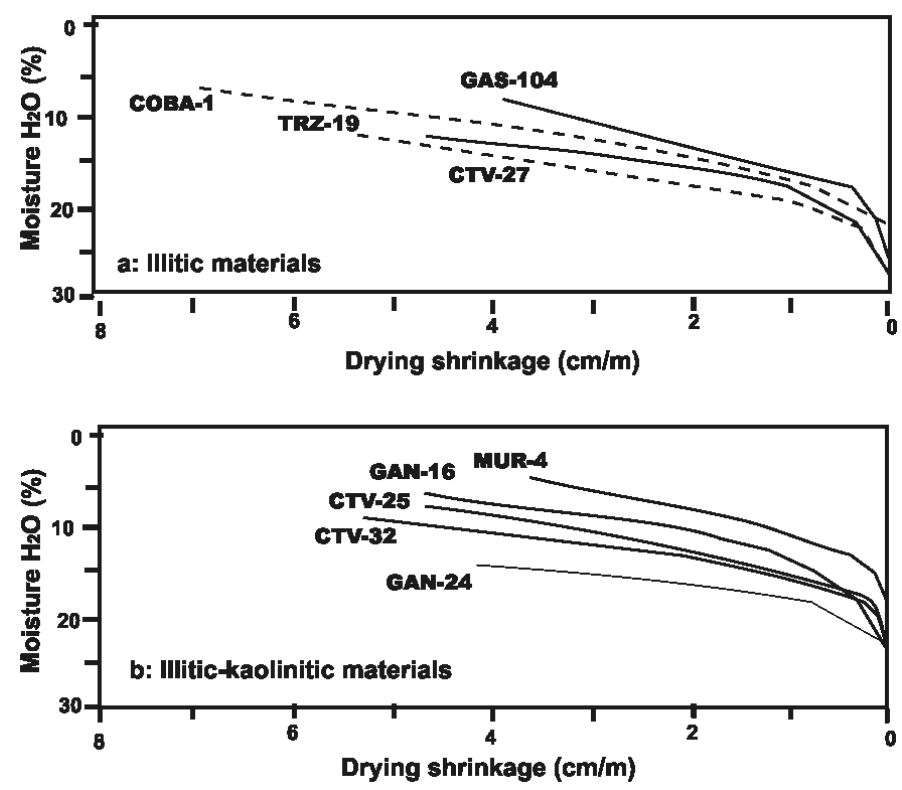

FIG. 4. Drying behaviour (Bigot's Curve).

$\left(21-36 \mathrm{~kg} / \mathrm{cm}^{2}\right)$. The worst bend strength (e.g. $6-15 \mathrm{~kg} / \mathrm{cm}^{2}$, TRZ-19 and MUR-4) depends on the small amount of phyllosilicates and the coarse particle-size distribution, while the very high resistance of COBA-1 $\left(87 \mathrm{~kg} / \mathrm{cm}^{2}\right)$ is due to its high clay minerals content.

Firing behaviour. Thermodilatometric curves are reproduced in Fig. 5. All samples present a similar expansion $(1-1.5 \%)$ until reaching $900^{\circ} \mathrm{C}$, followed by shrinkage up to the maximum tested temperature $\left(1000^{\circ} \mathrm{C}\right)$. During firing it was noticed that the $\alpha$-quartz was transformed into $\beta$-quartz, triggering a sudden expansion (point A, Fig. 5), while the opposite occurred (point D) during cooling. The COBA-1 sample presented a distinctive behaviour, with the greatest shrinkage values (4\%) beginning

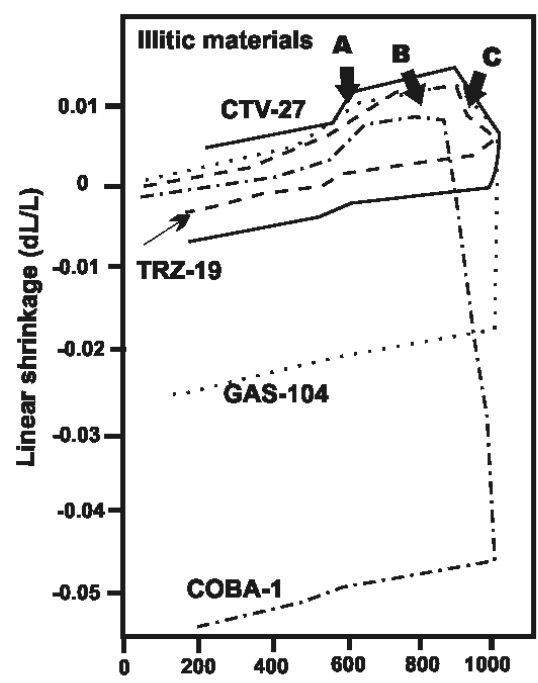

Firing temperature $\left({ }^{\circ} \mathrm{C}\right)$

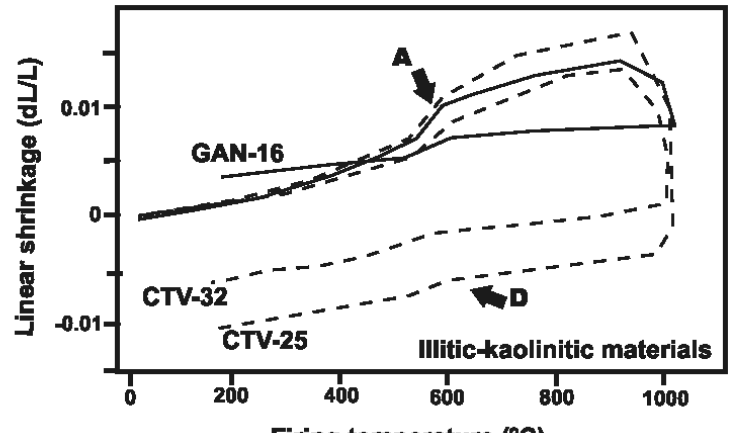

Firing temperature $\left({ }^{\circ} \mathrm{C}\right)$
A: $\alpha-Q t z$ transforms to $\beta-Q t z$
B: The contraction of sample COBA-1 begins
C: Secondary expansion of sample MON-23 begins D: $\beta$-Qtz transforms to $\alpha$-Qtz

Fig. 5. Dilatometric curves for materials. 
at a lower temperature $\left(\sim 850^{\circ} \mathrm{C}\right.$, point $\left.\mathrm{B}\right)$, while the MON-23 sample (containing $25 \%$ calcite) underwent a $0.6 \%$ shrinkage in the $875-950^{\circ} \mathrm{C}$ range, followed by a further dilatation $(0.5 \%)$ up to $1000^{\circ} \mathrm{C}$ (point $\mathrm{C}$ ).

Utilizing the results of the dilatometric test, an optimum firing temperature can be established: it must be at least $900^{\circ} \mathrm{C}$, because shrinkage of the pieces begins around this value. Higher temperatures should be adjusted to the ceramic properties, such as water absorption and mechanical resistance. Firing was carried out at 900,950 and $1000^{\circ} \mathrm{C}$, and water absorption (AA), linear shrinkage (CL), mechanical resistance, open porosity and apparent density were determined (Table 5).

The water absorption and linear shrinkage values have been represented graphically in the vitrification diagrams (Fig. 6) in order to observe the evolution of the ceramic test bodies in line with temperature change. As the firing temperature increased there was a general tendency for an increase in linear shrinkage and a reduction in water absorption, as well as an increase in bulk density and a decrease in the pore size.

The illitic clays' water absorption values range between 9 and $20 \%$, with shrinkages varying between 1 and $3.5 \%$. Noteworthy are the extreme values shown by the COBA-1 $(<8 \%$ water absorption, and linear shrinkage between 2.4 and $3.9 \%$ ) and MON-23 samples, the water absorption values of which are within the range $26-28 \%$, and the linear shrinkage values of which are between 1.1 and $0.6 \%$. The illitic-kaolinitic samples present average water absorption and linear shrinkage values between 10 and $23 \%$, and 0.8 and $2.3 \%$, respectively. It can be observed that, in general terms, the illitic clays present smaller values for water absorption and greater values for linear shrinkage.

The mechanical resistance increased noticeably as the firing temperature increased, presenting results of $46-206 \mathrm{~kg} / \mathrm{cm}^{2}$ for the illitic clays and $40-142 \mathrm{~kg} / \mathrm{cm}^{2}$ for the illitic-kaolinitic clays (Table 5).

Most results of colour measurements $\left(\mathrm{L}^{*}, \mathrm{a}^{*}\right.$ and $\mathrm{b}^{*}$ ) correspond to reddish shades (Table 6). As the firing temperature increased above $950^{\circ} \mathrm{C}$, the three parameters $\mathrm{L}^{*}, \mathrm{a}^{*}$ and $\mathrm{b}^{*}$ decreased steadily, as the pieces began to darken, due to the formation of hematite after the $\mathrm{Fe}$ oxide contained in the clay minerals. A different path is taken when there is a low $\mathrm{Fe}$ content and a large amount of carbonates.
Using the XRPD patterns of the fired samples (Fig. 7) it can be observed that at $900^{\circ} \mathrm{C}$ the following minerals were present: illite, quartz, calcite, K-feldspar, plagioclase and hematite (except GAN-16). All these minerals were present up to $1000^{\circ} \mathrm{C}$, with the exception of illite, calcite and dolomite, which gave rise to gehlenite, wollastonite and anorthite, which began to form at $900^{\circ} \mathrm{C}$. Moreover, the amount of hematite increased with the temperature.

\section{DISCUSSION}

The mineralogical composition is, according to Palmonari and Nassetti (1993) and Enrique Navarro and Amorós Albaro (1985), ideal for the manufacture of porous ceramic products.

The chemical analysis demonstrated that the high silica, alumina and potash contents are associated with the illitic nature of these clays. When $\mathrm{CaO}$ and $\mathrm{MgO}$ are abundant, they are associated with the high carbonate (calcite and dolomite) contents found in some samples.

Two groups of raw materials, one illitic and the other illitic-kaolinitic (with kaolinite $>15 \%$ ), were identified on the basis of both the mineralogical and chemical compositions of the fine fraction.

The results of the granulometric measurements (Table 3) indicate that samples of both groups: (1) are classified as loams and clayey loams (Shepard, 1954); (2) are rich in fine fraction $(<2 \mu \mathrm{m})$; and (3) present a suitable grain-size distribution for use as raw material in the building ceramics industry (Ferrandis et al., 1974).

The reason for this suitability is that the granulometric heterogeneity favours the packing of the fine particles in the spaces left by the larger particles. Sample COBA-1 is classified as loamy clay since its grain size is concentrated in the $<2 \mu \mathrm{m}$ fraction, a factor which affects its technological behaviour. Sample TRZ-19 is also noteworthy due to its low $<2 \mu \mathrm{m}$-fraction content, leading to inadequate particle packing.

According to Winkler's diagram shown in Fig. 8, most samples are located within, or very close to, the field for the production of large-format ceramic pieces.

During the drying process, two different stages could be established in all of the samples. In the first stage, most water utilized in the moulding process was lost through surface evaporation until they reached the leatherhard stage, corresponding to 
R. Artigas et al.

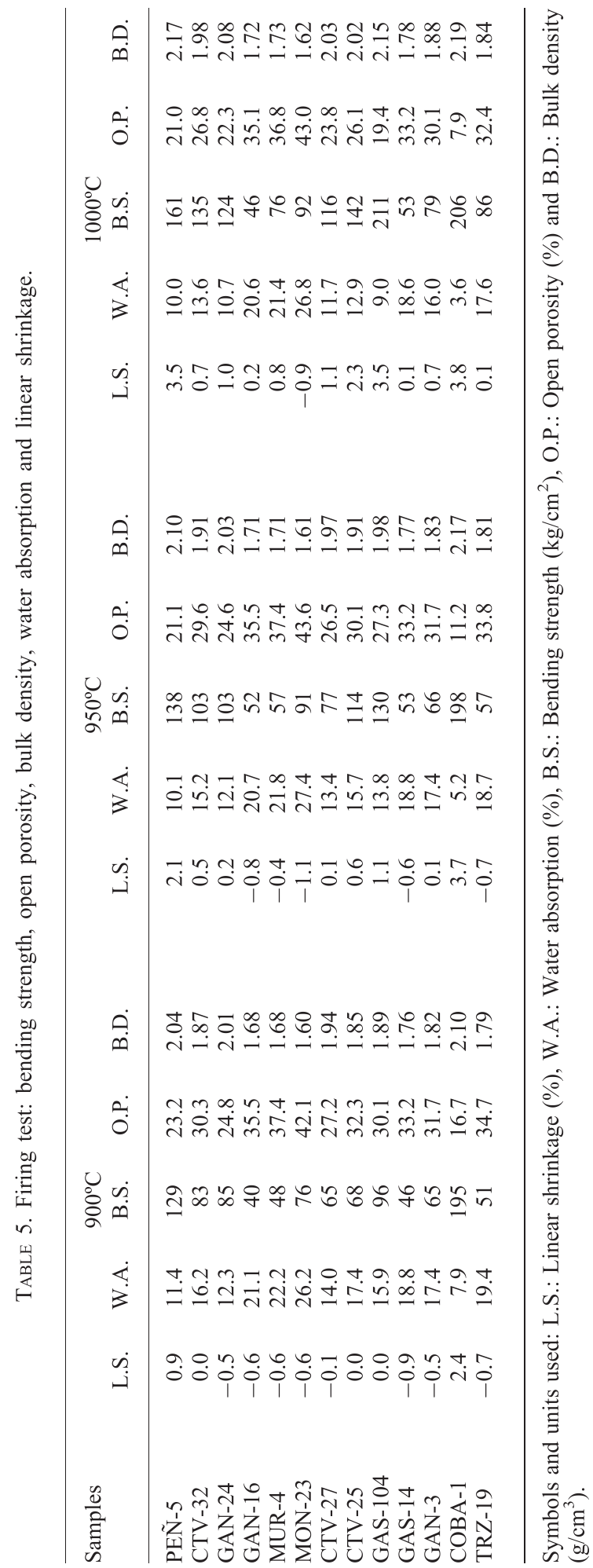




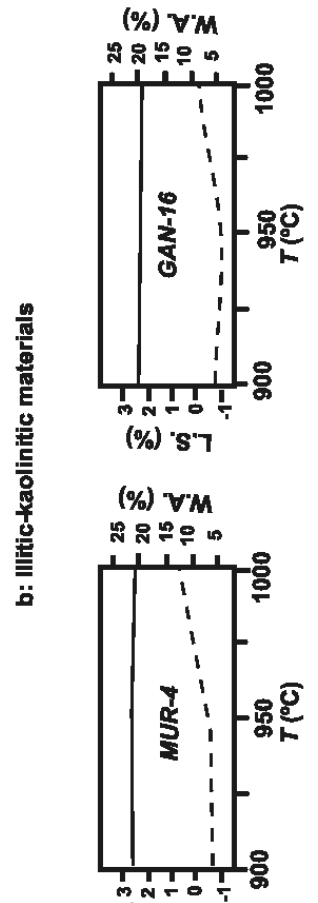

(\%) 's'

(\%) $\nabla \cdot M$

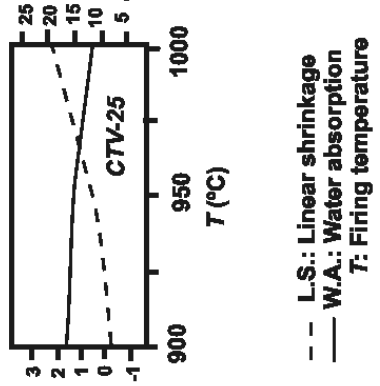

(\%) 's'

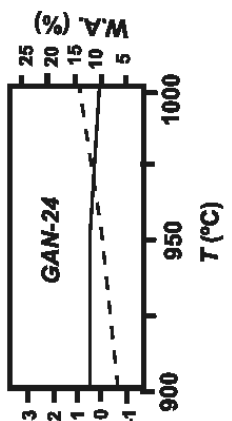

(\%) 's'
(\%) $\nabla M$

ํำ은웅

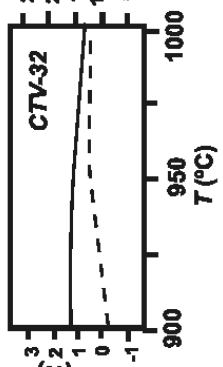

(\%)
(\%) $\forall ' M$

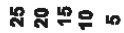

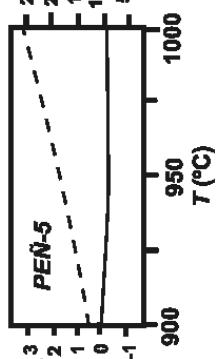

(\%) 's'7

(\%) $\nabla \cdot M$

논ำㄴำ은

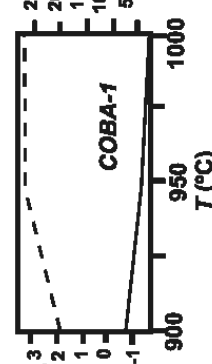

(\%)
(\%) $\mathbf{Y M}$

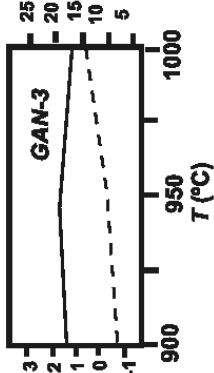

(\%)

(\%) $\nabla M$

ำ 준은

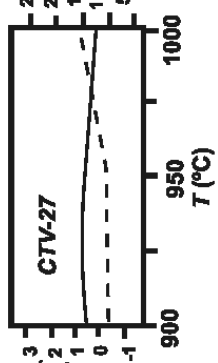

(\%) 's
(\%) $\forall M$

농 유 또우

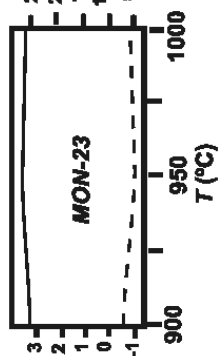

(\%) 's'

(\%) $\forall \cdot M$

농ำ 누운

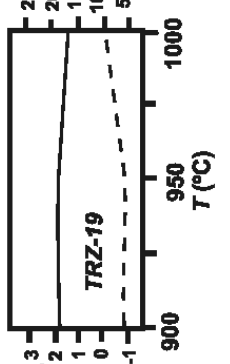

(\%)
(\%) $\forall M$

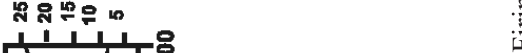


TABLE 6. Colour analysis with the parameters $L^{*}, a^{*}$ and $b^{*}$.

\begin{tabular}{|c|c|c|c|c|c|c|c|c|c|c|c|c|}
\hline \multirow[b]{3}{*}{ Samples } & \multicolumn{12}{|c|}{ Temperature $\left({ }^{\circ} \mathrm{C}\right)$} \\
\hline & \multicolumn{3}{|c|}{ Dry } & \multicolumn{3}{|c|}{900} & \multicolumn{3}{|c|}{950} & \multicolumn{3}{|c|}{1000} \\
\hline & $\mathrm{L}^{*}$ & $a^{*}$ & $b^{*}$ & $\mathrm{~L}^{*}$ & $a^{*}$ & $b^{*}$ & $\mathrm{~L}^{*}$ & $a^{*}$ & $b^{*}$ & $\mathrm{~L}^{*}$ & $a^{*}$ & $b^{*}$ \\
\hline PEÑ-5 & 58.23 & 15.68 & 15.90 & 50.92 & 26.21 & 25.10 & 49.37 & 26.63 & 23.76 & 45.08 & 24.49 & 19.86 \\
\hline CTV-32 & 49.98 & 13.24 & 13.76 & 57.37 & 24.80 & 22.90 & 56.78 & 25.93 & 21.64 & 53.38 & 22.50 & 19.63 \\
\hline GAN-24 & 50.45 & 15.57 & 17.32 & 56.88 & 26.93 & 24.09 & 54.34 & 28.95 & 25.46 & 51.38 & 27.19 & 22.13 \\
\hline GAN-16 & 77.58 & 2.87 & 13.38 & 76.01 & 13.54 & 14.29 & 76.86 & 10.82 & 15.89 & 78.05 & 11.52 & 17.05 \\
\hline MUR-4 & 58.04 & 14.50 & 20.92 & 59.43 & 24.05 & 23.56 & 59.53 & 25.94 & 24.43 & 57.66 & 24.93 & 23.65 \\
\hline MON-23 & 50.45 & 14.52 & 15.93 & 50.45 & 21.76 & 18.46 & 50.69 & 21.19 & 18.58 & 57.66 & 14.21 & 12.22 \\
\hline CTV-27 & 49.98 & 13.93 & 15.11 & 57.17 & 24.47 & 22.56 & 55.18 & 26.60 & 23.26 & 52.40 & 23.69 & 21.82 \\
\hline CTV-25 & 46.31 & 13.07 & 13.09 & 54.24 & 23.72 & 20.84 & 54.24 & 21.95 & 20.31 & 51.15 & 21.44 & 17.69 \\
\hline GAS-104 & 57.85 & 11.00 & 19.66 & 56.29 & 26.68 & 25.99 & 55.99 & 28.08 & 27.43 & 50.57 & 27.38 & 24.49 \\
\hline GAS-14 & 56.39 & 9.76 & 12.10 & 57.37 & 24.08 & 23.40 & 57.37 & 24.90 & 24.67 & 56.88 & 22.58 & 19.63 \\
\hline GAN-3 & 49.98 & 12.86 & 13.76 & 53.17 & 25.20 & 22.28 & 53.49 & 24.84 & 22.56 & 49.98 & 24.21 & 20.01 \\
\hline COBA-1 & 58.41 & 16.12 & 18.83 & 48.26 & 26.97 & 23.91 & 47.37 & 23.37 & 19.30 & 47.11 & 21.06 & 17.23 \\
\hline TRZ-19 & 51.04 & 13.11 & 12.48 & 50.57 & 22.05 & 18.38 & 54.24 & 21.87 & 16.28 & 52.62 & 21.78 & 16.23 \\
\hline
\end{tabular}

a humidity content in the $10-15 \%$ range. Once this level was reached, the second stage began, the process slowing down because it now involved a falling rate of evaporation from the interior of the test ceramic bodies. This was also the moment when the drying process could be accelerated without encountering shrinkage problems. The illitic-kaolinitic samples lost the working moisture
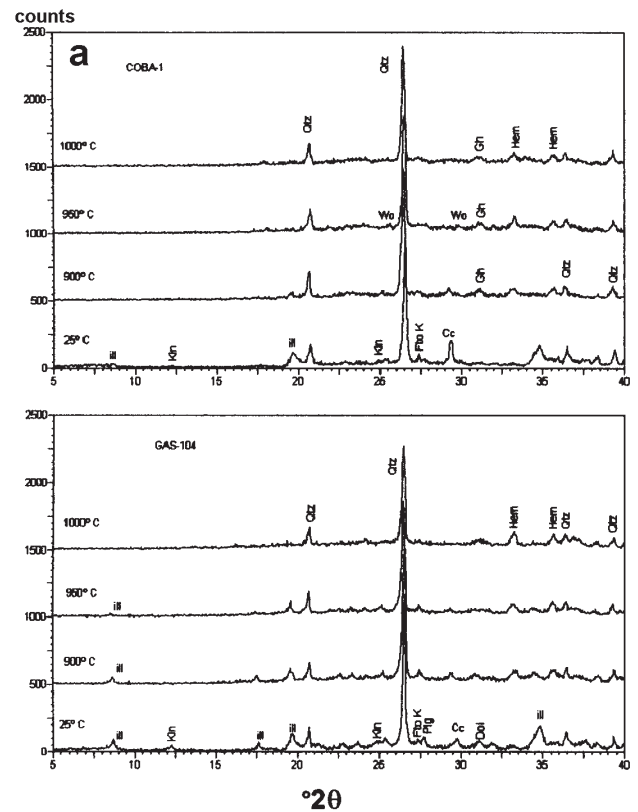

FIG. 7. XRD patterns for high-temperature minerals, (a) for illitic materials; (b) for illitic-kaolinitic materials. more quickly due to the low activity, meaning that this group of samples has no water-retaining qualities due to the system's high level of permeability.

In general terms, the illitic-kaolinite clays offer slightly less mechanical resistance when dry (Table 5) than the illitic clays. Both groups fulfil the drying-parameter requirements established by
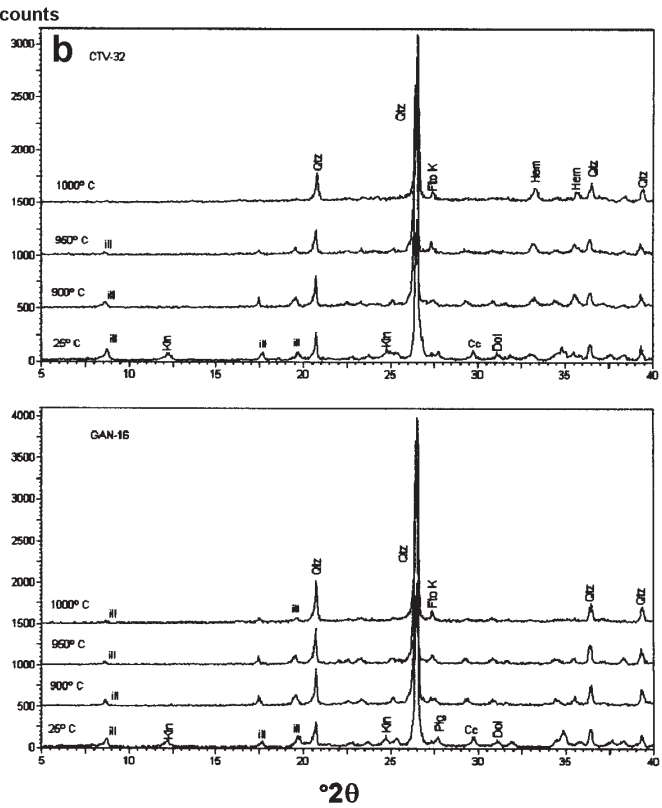


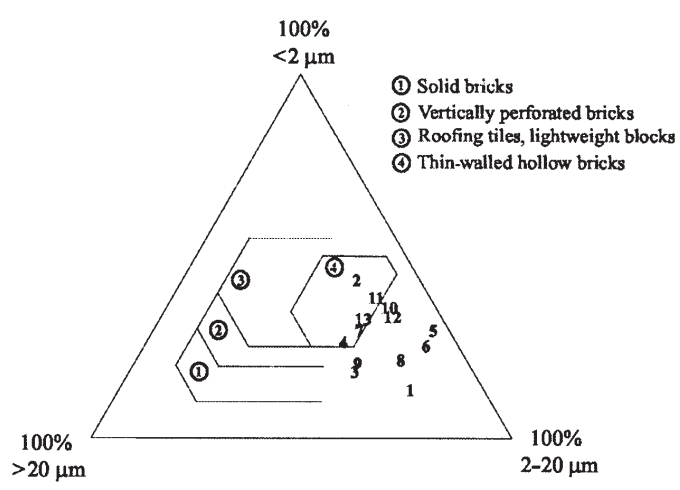

Fig. 8. Winkler (1954) diagram for the technological classification of bodies for structural clay products.

Dondi et al. (1998a,b). Exceptions to the above are samples TRZ-19, which, due to its low $<2 \mu \mathrm{m}$ fraction content and low (15\%) phyllosilicate content, presents a very small mechanical resistance $\left(6 \mathrm{~kg} / \mathrm{cm}^{2}\right)$; and GAN-16 and MUR-4 (with 12 and $15 \mathrm{~kg} / \mathrm{cm}^{2}$, respectively), due to their mineralogical composition which includes excessive proportions of quartz and kaolinite. Special mention must also be made of sample COBA-1 which has the greatest mechanical resistance and smallest shrinkage values, due to its high phyllosilicate content (the interstratified illite-smectite content, although relatively small, is sufficient to increase the values of the properties referred to above), and to its high fine-fraction content.

The hygroscopicity data indicate that neither group of raw materials absorbs $>1.5 \%$ of water, an acceptable figure for behaviour during firing. Only sample COBA-1 exceeds this value due to its mineralogical composition characterized by $5 \%$ of interstratified illite-smectite.

It was observed from the dilatometric test that all of the samples presented a progressive expansion up to $900^{\circ} \mathrm{C}$, followed by shrinkage until $1000^{\circ} \mathrm{C}$, triggered by the structural modifications of the preexisting minerals. These results coincide with those presented by Munier and Meneret (1950) for illitic clays. Shrinkage in sample COBA-1 is greatest due to its mineralogical composition and granulometric distribution, while the greatest expansion was found in MON-23 starting at $950^{\circ} \mathrm{C}$ due to the liberation of gases produced by the decomposition of $\mathrm{CaCO}_{3}$ into $\mathrm{CaO}$ and $\mathrm{CO}_{2}$. The onset of most illitickaolinitic clay shrinkage processes was displaced to higher temperatures due to the refractory nature of kaolinite (González-García et al., 1990; Kapur et al., 1995). In some cases, in those samples showing the highest kaolinite contents, shrinkage could begin at $925^{\circ} \mathrm{C}$ (MUR-4 and GAN-16).

During firing it was observed that water absorption and open porosity were reduced as the temperature increased. However, the measured values are relatively high $(>10 \%)$, meaning that these firing temperatures are unsuitable for manufacturing vitrified products. Only sample COBA-1 gives dense products, though with a high shrinkage value $(>3 \%)$. Moreover, in the test samples manufactured with this material, small white grains, or 'caliche', were produced. This defect is associated with the presence of coarse grains of calcite $(>1 \mathrm{~mm})$ which are unable to react completely during firing and undergo a hydration and carbonatation during cooling.

In the study of the high-temperature crystalline phases, the disappearance of the most abundant minerals (illite, calcite, feldspars and dolomite) was observed together with an increase in hematite content (triggering the appearance of a more intense red colour) and the formation of silicates such as wollastonite, gehlenite and anorthite (Peters and Iberg, 1978; Dunham et al., 1992; Cultrone et al., 2001), as a result of recombining the alumina and silica with $\mathrm{CaO}$ coming from the decomposition of the carbonates. These newly formed crystalline phases increased the mechanical resistance of the ceramic test bodies.

The variations in the technological parameters were less pronounced in illitic-kaolinitic raw materials due to the fact that they present smaller proportions of fusible minerals, with respect to illitic raw materials. Due to its high $\mathrm{K}_{2} \mathrm{O}$ content, the melting point of the ceramic pieces made of the illitic materials is correspondingly lower. There are however samples that do not behave in this way, e.g. COBA-1, MON-23, TRZ-19, GAN-16 and MUR-4.

In summary, and taking into account the water absorption, bending strength, linear shrinkage, open porosity and apparent density requirements as described by Dondi et al. (1998a,b), the studied samples were found suitable for use as raw materials in the manufacture of porous and redcoloured ceramic building elements if fired at temperatures of $\sim 950-1000^{\circ} \mathrm{C}$. There are, however, exceptions (TRZ-19, GAN-16 and MUR-4) among the studied raw materials, which present a low mechanical resistance during both the drying and firing processes. The reason for this 
behaviour is to be found in their granulometry (TRZ-19) and composition (high quartz and kaolinite contents - GAN-16 and MUR-4).

\section{CONCLUSIONS}

In the Cameros Basin, extensive outcrops expose thick deposits of lutite materials within the SD-5, 6 and 7 siliciclastic formations. In most units, the mineralogical composition is highly homogeneous, showing small variations that can be attributed to carbonates, illite and kaolinite. The samples selected for the technological study are distinguished into two groups, one illitic and the other illitic-kaolinitic ( $>15 \%$ kaolinite).

The laboratory simulation of the brickmaking process demonstrated that these materials can be utilized in the manufacture of red-coloured ceramic building elements. The best firing temperature is between 950 and $1000^{\circ} \mathrm{C}$. The raw materials TRZ-19, GAN-16 and MUR-4 are unsuitable due to their low mechanical resistance, due to their coarse-grained particle distribution and/or mineralogy.

The samples CTV-25, CTV-27, CTV-32, GAN24 and GAS-104 are those which may be fired at higher temperatures, as their mechanical resistance increases while their water absorption decreases, thus probably making them suitable materials for manufacturing vitrified products.

\section{ACKNOWLEDGMENTS}

This work was carried out thanks to the DIGICYT (Project number PB97-0298). The authors would like to thank Ms Guia Guarini (Istituto di Scienza e Tecnologia dei Materiali Ceramici, Faenza, Italy) and Mr Carlos Rivera Cabanillas (Laboratory of Applied Mineralogy, UCLM), for their collaboration in this project. The authors are grateful to C. Gomes, D.G.F. Long and J. Jiménez for their useful suggestions and improvement of the text.

\section{REFERENCES}

Alonso-Azcárate J., Barrenechea J., Rodas M. \& Mas J. R. (1995) Comparative study of the transition between very low-grade and low-grade metamorphism in siliciclastic and carbonate sediments; Early Cretaceous, Cameros Basin (northern Spain). Clay Minerals, 30, 407-419.

Arribas J., Alonso A., Mas R. \& Tortosa A. (2001) The role of the structural Hercynian units on the petrogenesis of siliciclastic deposits from the wes- tern Cameros Basin (Late Jurassic-Early Cretaceous Iberian Rift), North Spain. International Association of Sedimentologists, $21^{\text {st }}$ Meeting, pp. 119-120.

Arribas J., Alonso A., Mas R., Tortosa A., Rodas M., Barrenechea J.F., Alonso-Azcárate J. \& Artigas R. (2003) Sandstone petrography of continental depositional sequences of an intraplate rift basin: western Cameros basin (North Spain). Journal of Sedimentary Research, 73, 307-325.

Bain J.A. (1968) A plasticity chart as an aid to the identification and assessment of industrial clays. Clay Minerals, 9, 1-17.

Barrenechea J., Rodas M. \& Mas J.R. (1995) Clay mineral variations associated with diagenesis and low-grade metamorphism of Early Cretaceous sediments in the Cameros Basin, Spain. Clay Minerals, 30, 119-133.

Casquet C., Galindo C., González-Casado J.M., Alonso A., Mas R., Rodas M., García E. \& Barrenechea J.F. (1992) El metamorfismo en la cuenca de los Cameros; geocronología e implicaciones tectónicas. Geogaceta, 11, 22-25.

Cultrone G., Rodríguez-Navarro C., Sebastián E., Cazalla O. \& De la Torre M.J. (2001) Carbonate and silicate phase reactions during ceramic firing. European Journal of Mineralogy, 13, 621-634.

Dondi M., Marsigli M. \& Venturi I. (1998a) Sensibilità allssiccamento e caratteristiche porosimetriche delle argille italiane per laterizi. Ceramurgia, XXVIII, $1-8$.

Dondi M., Marsigli M. \& Venturi I. (1998b) Technological requirements of raw materials for heavy clay products. Proceedings of the $2^{\text {nd }}$ Mediterranean Clay Meeting, Aveiro, Portugal, 2, pp. 204-207.

Dunham A.C., McKnight A.S. \& Warren I. (1992) The determination and application of time-temperaturetransformation diagrams for brick, tile and pipe clays. Final Report to the Mineral Industry Research Organisation of Project RC56: TTT diagrams for Brick, Tile and Pipe Clays.

Enrique Navarro J.E. \& Amorós Albaro J.L. (1985) Tecnología Cerámica. Vol. I. Introducción a la Tecnología Cerámica. Materias primas cerámicas. Instituto de Química Técnica. Universidad de Valencia, Spain, 155 pp.

Ferrandis V.A., González Peña J.M ${ }^{\mathrm{a}}$. \& Sandoval del Río F. (1974) Estudio fisicoquímico y tecnológico de un grupo de arcillas para ladrillería. Boletín de la Sociedad Española de Cerámica y Vidrio, 13, 495-504.

González-García F., Romero-Acosta V., García-Ramos G. \& González-Rodríguez M. (1990) Firing transformations of mixtures of clays containing illite, kaolinite and calcium carbonate utilised by ornamental tile industries. Applied Clay Science, 5, $361-375$. 
Guimera J., Alonso A. \& Mas J.R. (1995) Inversion of an extensional-ramp basin by a newly formed thrust; the Cameros Basin (N. Spain). Pp. 433-453 in: Basin Inversion (J.G. Buchanan and P.G. Buchanan, editors). Special Publication 88, Geological Society of London.

Kapur S., Sakarya N., Karaman C., FitzPatrick E.A. \& Pagliani M. (1995) Micromorphology of basaltic ceramics. British Ceramic Transactions, 94, 33-37.

Kretz R. (1983) Symbols for rock-forming minerals. American Mineralogist, 68, 277-279.

Martín-Closas C. \& Alonso-Millán A. (1998) Estratigrafía y bioestratigrafía (Charophyta) del Cretácico Inferior en el sector occidental de la Cameros Basin (Cordillera Ibérica). Revista de la Sociedad Geológica de España, 11, 253-269.

Mas J.R., Alonso A. \& Guimerá J. (1993) Evolución tectonosedimentaria de una cuenca extensional intraplaca: La cuenca finijurásica-eocretácica de Los Cameros (La Rioja-Soria). Revista de la Sociedad Geológica de España, 6, 129-144.

Moore D.M. \& Reynolds R.L. (1989) X-ray Diffraction and the Identification and Analysis of Clay Minerals. Oxford University Press, New York, 332 pp.

Munier P. \& Meneret J. (1950) Identification des argiles cerámiques pour le dilatométrie en cru. Bulletin de la Société française de Céramique, 7, 6-17.

NORMA U.N.E. 67027 (1984) Ladrillos de arcilla cocida. Determinación de la absorción de agua.

NORMA U.N.E. 67042 (1988) Piezas cerámicas de arcilla cocida de gran formato. Determinación de la resistencia a la flexión.

NORMA U.N.E. 103-104 (1993) Determinación del límite plástico de un suelo.
NORMA U.N.E. 103-103 (1994) Determinación del límite líquido de un suelo por el método del aparato de Casagrande.

NORMA U.N.E. 103-101 (1995) Análisis granulométrico de suelos por tamizado.

Palmonari C. \& Nassetti G. (1993) Traditional ceramics. Evolution in the last decade and future trends. Third Euro-ceramics Conference, 2 (P. Durán and J.F. Fernández, editors). Faenza Editrice, Italy, pp. 879-891.

Peters T. \& Iberg R. (1978) Mineralogical changes during firing of calcium-rich brick clays. American Ceramic Society Bulletin, 57, 503-509.

Salas R., Guimerá J., Mas R., Martín Closas C., Meléndez A. \& Alonso A. (2001) Evolution of the Mesozoic Central Iberian Rift System and its Cenozoic inversion (Iberian Chain). Pp. 145-186 in: Peri-Tethyan Rift/Wrench Basins and Passive Margins (P.A. Ziegler, W. Cavazza, A.H.F. Robertson and S. Crasquin-Soleau, editors). Muséum National D'Histoire Naturelle, Mèmories, I, 186. Peri-Tethys Memoir 6.

Schultz L.G. (1964) Quantitative interpretation of mineralogical composition from X-ray and chemical data for the Pierra shale. US Geological Survey Professional Paper, 391-c, 1-31.

Shepard F.P. (1954) Nomenclature based on sand-siltclay ratios. Journal of Sedimentary Petrography, 24, $151-158$.

Winkler H.G.F. (1954) 'Bedeutung der Korngrössenverteilung und des Mineral-bestandes von Tonen für die Herstellung grobkeramischer Erzeugnisse'. Berichte der Deutschen Keramischen Gesellschaft, 31, 337-343. 